\title{
CARACTERÍSTICAS FÍSICAS, QUÍMICAS E ORGANOLÉPTICAS DE QUIPÁ (Tacinga inamoena, Cactaceae) ${ }^{1}$
}

\author{
ANDRÉA CARLA MENDONÇA DE SOUZA², GUILLERMO GAMARRA-ROJAS ${ }^{3}$, \\ SAMARA ALVACHIAN CARDOSO ANDRADE ${ }^{4}$, NONETE BARBOSA GUERRA ${ }^{5}$
}

RESUMO- A Opuntia ficus-indica tem-se destacado como principal produtora de frutos e forragens, motivo pelo qual tem sido bastante pesquisada. Além desta espécie, no semi-árido do Nordeste é encontrada a Tacinga inamoena, planta nativa, cujo fruto, embora também utilizado pelo agricultor como alternativa alimentar, não foi objeto de nenhuma pesquisa até o momento, justificando este trabalho para avaliar seu potencial nutricional e industrial, por meio das características organolépticas, químicas e físicas, conteúdo nutricional e composição mineral. O quipá apresenta características organolépticas similares às de frutos de mesmo gênero, com rendimento da porção comestível, polpa e pericarpo carnoso de $62,87 \%$ do peso total do fruto. No que diz respeito à composição química, a polpa difere significativamente do pericarpo carnoso, com superioridade deste último, que apresenta maior teor de minerais, destacando-se dentre estes o cálcio, o magnésio e o potássio que apresentaram valores de 587,04mg, 257,02mg e 318,01mg, respectivamente. Os resultados evidenciam que o quipá é adequado para consumo in natura e apresenta potencial para aproveitamento industrial.

Termos para indexação: caracterização de frutos, planta nativa, rendimento.

\section{PHYSICAL CHEMICAL AND CHEMICALCHARACTERISTICS OF 'QUIPÁ' (Tacinga inamoena, Cactaceae)}

\begin{abstract}
Provided that the Opuntia ficus-indica is the main producer of fruit and forages, it has been the object of extensive research. Besides, another species Tacinga inamoena, native plant, which its fruit has been used as an alternative food source can also be found in the semi-arid northeastern region of Brazil. However, this native plant had not been the object of any research yet. The aim of this work is to evaluate its industrial and nutritional potential through the organoleptic, physical, chemical characteristics, nutritional content and mineral composition. The Quípa shows organoleptic characteristics similar to other fruit of the same gender. Its edible portion, pulp and fleshy pericarp, corresponds to $62,8 \%$ of the total fruit weight. As regard to chemical composition, the pulp differs significantly from the fleshy pericarp, the latter showing higher content of minerals among them calcium, magnesium and potassium with values of $587,04 \mathrm{mg}, 257,02 \mathrm{mg}$ and $318,01 \mathrm{mg}$, respectively. The results showed that Quípa is appropriate for consumption in natura and shows industrial potential.
\end{abstract}

Index terms: Characterizing of fruits, native plant, revenue.

\section{INTRODUÇÃO}

A O. ficus-indica tem-se destacado como a principal cactácea produtora de frutos e forragem, sendo também a mais estudada, utilizada e difundida nas regiões semi-áridas do planeta. No Brasil, o cultivo para a produção de frutos concentra-se no Estado de São Paulo. Do total, uma pequena parte é destinada ao mercado interno, e a maior parcela é exportada para a Europa e Estados Unidos, onde existe o hábito de consumo do fruto (Garcia e Valdez, 2003; Glass, 2005). A valorização dos frutos de O. ficusindica nos mercados nacional e internacional abre perspectivas para outras cactáceas regionais, como o quipá, cuja comercialização contribuiria para melhorar as condições de vida da população do semi-árido nordestino.

O quipá [Tacinga inamoena (K. Schum.) N. P. Taylor e Stuppy (=Opuntia inamoena)], espécie popularmente conhecida como cumbeba ou gogóia, é uma planta nativa da região Nordeste e encontra-se distribuída em quase todo o semi-árido. Seus frutos são do tipo baga ovóide a subgloboso, 3,0-4,0 x 2,4-3,5 cm de diâmetro longitudinal e transversal, respectivamente, variando do amarelo ao laranja fosco, com porção basal avermelhada ou toda vermelha, fosca; câmara seminífera ocupando quase todo o espaço interno, preenchido por massa carnosa, cor de pêssego clara, constituída pelos funículos das sementes (polpa). Estas são abundantes e submersas na massa carnosa dos funículos, lenticulares, castanho-claro, de bordo mais claro; envolvidas pelo arilóide fibro-carnoso (Andrade Lima, 1989).

Os frutos e cladódios têm sido utilizados na zona rural para alimentação animal, enquanto, na alimentação humana, apenas em situações de escassez com a finalidade de complementar a alimentação ou, em muitos casos, como a única opção de alimento. Estas constatações despertaram grande interesse da comunidade

(Trabalho 119-06). Recebido em 16-08-2006. Aceito para publicação em : 19-04-2007.

${ }^{2}$ Mestranda da Pós-Graduação em Nutrição da UFPE, Recife-PE; E-mail: acmsnutri@uol.com.br

${ }^{3}$ Agrônomo, Dr.,Consultor autônomo. E-mail: ggamarra@terra.com.br

${ }^{4}$ Professora do Depto. de Engenharia Química da UFPE. Recife - PE. E-mail: samaraandrade@uol.com.br

${ }^{5}$ Professora Titular do Depto. de Nutrição da UFPE. Recife-PE. E-mail: leaal@nuticao.ufpe.br 
científica em desvendar o potencial nutricional e comercial de frutos nativos de espécies subutilizadas, como o quipá, ( $T$. inamoena) quanto às suas características físicas, químicas e organolépticas.

\section{MATERIAL E MÉTODOS}

Constituído de três lotes, de 30 frutos de Tacinga inamoena cada, colhidos em 04-08-04, 02-10-04 e 05-01-05, tendo como critério a coloração da casca completamente amarela e amarelo-alaranjada, segundo a experiência local, pelos agricultores do Pólo Sindical da Borborema, na região de Cariri-Curimataú-PB. Os frutos, em estádio de maturação plena, foram transportados para o Laboratório de Experimentação e Análises de Alimentos Nonete Barbosa Guerra (LEAAL) do Departamento de Nutrição da Universidade Federal de Pernambuco (UFPE), onde foram processados e analisados. As características organolépticas (cor, aparência, aroma, sabor, textura e consistência) foram avaliadas por seis analistas do LEAAL, de acordo com o Instituto Adolfo Lutz (1985). A cada analista, foram oferecidos dois frutos de cada lote, um inteiro e um cortado ao meio no sentido transversal. No laboratório, os demais frutos foram lavados em água corrente e submetidos à abrasão, para a retirada dos gloquídeos, secados, pesados e medidos individualmente. Em seguida, as cutículas (denominadas cascas) foram retiradas e pesadas, e os frutos foram fracionados em seus constituintes (pericarpo carnoso, polpa e sementes). Para a realização dos ensaios analíticos, conduzidos em triplicata, as porções comestíveis (pericarpo e polpa com sementes) foram homogeneizadas separadamente em miniprocessador, e armazenadas a $18^{\circ} \mathrm{C}$ negativos em freezer doméstico. Atributos físicos: peso dos frutos (g) e diâmetros ( $\mathrm{mm}$ ). Atributos químicos e composição nutricionais: umidade, cinzas, proteínas, lipídeos, fibra alimentar total (AOAC, 2002); sódio, zinco, potássio, cálcio, cobre, ferro, magnésio, por espectroscopia de absorção atômica e fosfato, por espectrofotômetro de absorção molecular (AOAC, 2002); carboidratos (Marais \& Erasmus, 1977) e valor calórico total (VCT); sólidos solúveis totais ( $\left.{ }^{\circ} \mathrm{Brix}\right), \mathrm{pH}$, acidez total titulável (\% ácido cítrico) (AOAC, 2002) e relação ${ }^{\circ}$ Brix/Acidez, carotenóides totais (Rodriguez-Amaya, 1999). Os dados foram submetidos ao teste " $\mathrm{t}$ " de student e à análise de variância (ANOVA), ambos ao nível de 5\% significância, através do programa "Statistica for Windows" 6.0 .

\section{RESULTADOS E DISCUSSÃO}

De modo geral, os descritores de aparência externa e interna (Tabela 1) ratificam Andrade Lima (1989). No que diz respeito ao aroma, "flavor", cor e textura, foram considerados satisfatórios, uma vez que não foi manifestada rejeição pelos analistas. Segundo Thé et al. (2001), estas características influenciam, de forma direta, na aceitação final do produto pelo consumidor. Comparando os atributos externos da T. inamoena com o descrito por Ramadan \& Mörsel (2003) e García \& Valdez (2003) para O. ficus-indica, confirma-se a semelhança no que se refere ao formato.

$\mathrm{Na}$ determinação das características físicas, foram considerados os aspectos que geralmente são utilizados para avaliar a uniformidade dos frutos, pela influência direta que exercem na seleção de variedades, tanto para fins industriais como para consumo in natura (Bicalho \& Penteado, 1981).

As diferenças entre os lotes (Tabela 2) decorrem da variabilidade genética presente em frutos silvestres como o quipá, que não passaram por processo de seleção. A forma, o peso e o tamanho dos frutos encontram-se em conformidade com o descrito por Andrade Lima (1989). Em relação aos seus constituintes, verificou-se que a porção carnosa, que abrange o pericarpo carnoso e a polpa com sementes, representa percentual de $62,87 \%$ do peso total do fruto. Ao estabelecer comparações entre os constituintes da T. inamoena e da O.ficus-indica (Figura 1), verifica-se que os frutos do quipá apresentam valores mais elevados de porção carnosa e menores quantidades de casca e de sementes. A última característica favorece a T. inamoena, uma vez que o elevado teor de sementes constitui um fator de redução da aceitabilidade do fruto, particularmente pelos consumidores não familiarizados com o seu consumo (Valdez, 2002; Nerd \& Mizrahi, 2001). Além disso, o percentual de porção carnosa (Figura 1) confere a este fruto considerável potencial como matéria-prima para a industrialização.

A polpa difere $(\mathrm{p}<0,05)$ do pericarpo carnoso quanto à acidez e $\mathrm{pH}$. $\mathrm{O}$ valor de $\mathrm{pH}$ encontra-se próximo da faixa considerada limite, 3,7 a 4,5, para o desenvolvimento de microrganismos (Baruffaldi \& Oliveira, 1998). A acidez e o pH da polpa diferem dos valores citados para O. ficus-indica $(0,12 ; 5,9)$, (0,06; 6,37), (0,06; 5,33) Coelho et al. (2004), Sepúlveda \& Sáenz (1990), Bicalho \& Penteado (1982), respectivamente. Em relação ao teor de sólidos solúveis totais, os valores determinados para a polpa de T. inamoena são inferiores aos descritos para O. ficusindica, por Sáenz (2000), Sepúlveda \& Sáenz (1990) e Bicalho \& Penteado (1982). A relação ${ }^{\circ}$ Brix/acidez: 15,88 na polpa e 19,50 no pericarpo carnoso indica a predominância da acidez sobre a doçura, corroborando a avaliação das características organolépticas descritas na Tabela 1 , ou seja, sabor suave, pouco doce.

No que diz respeito ao valor nutritivo, diferenças significantes foram observadas na Tabela 3 entre polpa e pericarpo carnoso, na maior parte das características analisadas. De modo geral, essas características encontram-se em consonância com os referidos para O. ficus-indica por Sepúlveda \& Sáenz (1990), Sawaya \& Khan (1982) e Bicalho \& Penteado (1981), corroborando a proximidade botânica entre as duas espécies. Os carotenóides, importantes pigmentos amplamente encontrados na natureza, alguns dos quais exercem também função de pró-vitamina A e de antioxidantes naturais (Rodriguez-Amaya, 1999), também foram encontrados em maior concentração no pericarpo carnoso $(3,37 \mathrm{mg} / 100 \mathrm{~g})$.

No que diz respeito aos minerais, os resultados corroboram Bicalho \& Penteado (1981), que consideram o O. ficus-indica, como importante fonte de cálcio $(38,91 \mathrm{mg} / 100 \mathrm{~g})$ e fósforo (30,36mg/100g), sendo que, para a espécie em estudo, também se destacam o magnésio e o potássio. O teor determinado no pericarpo carnoso aproxima-se dos valores encontrados para o

Rev. Bras. Frutic., Jaboticabal - SP, v. 29, n. 2, p. 292-295, Agosto 2007 
O. ficus-indica por Sáenz (2000), Sepúlveda \& Sáenz (1990) e Sawaya et al. (1983), sendo significativamente superiores aos relatados por Bicalho \& Penteado (1981).

TABELA 1 - Características organolépticas do fruto da $T$. inamoena

\begin{tabular}{ll}
\hline \multicolumn{1}{c}{ Externas } & \multicolumn{1}{c}{ Internas } \\
\hline & \\
- Formato arredondado & - Cor amarelo intenso, atrativa; \\
(globoso) & - Aparência agradável \\
- Aparência pouco atraente; & - Aroma suave, agradável; \\
- Cor rosa. & - Sabor suave, suculento, pouco \\
& doce; \\
& - Textura firme; \\
& - Consistência elástica, macia; \\
& - Aparente viscosidade. \\
\hline
\end{tabular}

TABELA 2 - Valores médios dos diâmetros, pesos do fruto, casca, pericarpo carnoso,polpa e sementes da $T$. inamoena.

\begin{tabular}{rccc}
\hline & & T. inamoena & \\
\hline Características Físicas & Lote 1 & Lote 2 & Lote 3 \\
\hline Diâmetro longitudinal $(\mathrm{mm})$ & $3,39 \pm 0,01^{\mathrm{a}}$ & $3,24 \pm 0,02^{\mathrm{b}}$ & $3,44 \pm 0,09^{\mathrm{a}}$ \\
Diâmetro transversal $(\mathrm{mm})$ & $3,06 \pm 0,01^{\mathrm{b}}$ & $2,91 \pm 0,01^{\mathrm{c}}$ & $3,15 \pm 0,05^{\mathrm{a}}$ \\
Peso do fruto $(\mathrm{g})$ & $12,50 \pm 0,06^{\mathrm{b}}$ & $12,59 \pm 0,01^{\mathrm{b}}$ & $13,00 \pm 0,50^{\mathrm{a}}$ \\
Peso das cascas $(\mathrm{g})$ & $3,85 \pm 0,10^{\mathrm{b}}$ & $3,80 \pm 0,05^{\mathrm{b}}$ & $4,27 \pm 0,02^{\mathrm{a}}$ \\
Peso do pericarpo $(\mathrm{g})$ & $5,27 \pm 0,04^{\mathrm{a}}$ & $5,03 \pm 0,03^{\mathrm{c}}$ & $5,15 \pm 0,05^{\mathrm{b}}$ \\
Peso da polpa $(\mathrm{g})$ & $2,61 \pm 0,03^{\mathrm{b}}$ & $3,10 \pm 0,05^{\mathrm{a}}$ & $2,78 \pm 0,18^{\mathrm{b}}$ \\
Peso das sementes $(\mathrm{g})$ & $0,75 \pm 0,06^{\mathrm{ac}}$ & $0,65 \pm 0,05^{\mathrm{bc}}$ & $0,79 \pm 0,04^{\mathrm{a}}$
\end{tabular}

${ }^{1}$ Valores representam médias ( \pm desvio-padrão), $n=30$ frutos.

Médias seguidas de letras iguais, na horizontal, não diferem significativamente, ao nível de 5\%, pelo teste de Duncan.

TABELA 3 - Características físicas e químicas e químicas da porção comestível de frutos de T. inamoena.

\begin{tabular}{lcc}
\hline & \multicolumn{2}{c}{ T. inamoena } \\
\cline { 2 - 3 } \multicolumn{1}{c}{ Características } & Polpa & Pericarpo \\
\hline pH & $3,72 \pm 0,05^{\mathrm{b}}$ & $4,74 \pm 0,0006^{\mathrm{a}}$ \\
Acidez (\% ácido cítrico) & $0,63 \pm 0,06^{\mathrm{a}}$ & $0,47 \pm 0,06^{\mathrm{b}}$ \\
Sólidos solúveis totais( ${ }^{\circ}$ Brix a $\left.20^{\circ} \mathrm{C}\right)$ & $9,00 \pm 0,01^{\mathrm{a}}$ & $10,00 \pm 0,01^{\mathrm{a}}$ \\
${ }^{\circ}$ Brix/Acidez & $15,88 \pm 1,37^{\mathrm{a}}$ & $19,50 \pm 2,60^{\mathrm{a}}$ \\
Umidade $(\mathrm{g} / 100 \mathrm{~g})$ & $88,69 \pm 0,11^{\mathrm{a}}$ & $89,67 \pm 0,82^{\mathrm{a}}$ \\
Cinzas $(\mathrm{g} / 100 \mathrm{~g})$ & $0,57 \pm 0,02^{\mathrm{b}}$ & $1,31 \pm 0,02^{\mathrm{a}}$ \\
Proténas $(\mathrm{g} / 100 \mathrm{~g})$ & $1,15 \pm 0,01^{\mathrm{a}}$ & $0,36 \pm 0,01^{\mathrm{b}}$ \\
Lipídios $(\mathrm{g} / 100 \mathrm{~g})$ & $0,21 \pm 0,01^{\mathrm{b}}$ & $0,26 \pm 0,02^{\mathrm{a}}$ \\
Carboidratos $(\mathrm{g} / 100 \mathrm{~g})$ & $9,38 \pm 0,15^{\mathrm{a}}$ & $8,42 \pm 0,85^{\mathrm{a}}$ \\
Fibras $(\mathrm{g} / 100 \mathrm{~g})$ & $0,06 \pm 0,01^{\mathrm{a}}$ & $0,07 \pm 0,01^{\mathrm{a}}$ \\
V.C.T. $(\mathrm{kcal} / 100 \mathrm{~g})$ & $44,05 \pm 0,44^{\mathrm{a}}$ & $37,43 \pm 3,2^{\mathrm{b}}$ \\
Carotenóides totais (mg/100g) & $0,47 \pm 0,02^{\mathrm{b}}$ & $3,37 \pm 0,02^{\mathrm{a}}$ \\
Cobre & $0,05 \pm 0,006^{\mathrm{a}}$ & $0,04 \pm 0,003^{\mathrm{b}}$ \\
Ferro & $0,72 \pm 0,007^{\mathrm{a}}$ & $0,51 \pm 0,009^{\mathrm{b}}$ \\
Zinco & $0,05 \pm 0,019^{\mathrm{b}}$ & $0,62 \pm 0,026^{\mathrm{a}}$ \\
Sódio & $1,38 \pm 0,266^{\mathrm{b}}$ & $2,03 \pm 0,212^{\mathrm{a}}$ \\
Magnésio & $64,24 \pm 2,088^{\mathrm{b}}$ & $257,02 \pm 10,124^{\mathrm{a}}$ \\
Potássio & $87,90 \pm 3,22^{\mathrm{b}}$ & $318,01 \pm 2,36^{\mathrm{a}}$ \\
Cálcio & $232,11 \pm 0,249^{\mathrm{b}}$ & $587,04 \pm 20,89^{\mathrm{a}}$ \\
Fósforo & $47,55 \pm 1,57^{\mathrm{a}}$ & $13,82 \pm 0,56^{\mathrm{b}}$ \\
\hline
\end{tabular}

${ }^{1}$ Valores representam médias ( \pm desvio-padrão), $n=30$ frutos.

Médias seguidas de letras iguais, na horizontal, não diferem significativamente, ao nível de 5\%, pelo teste "t" de Student.

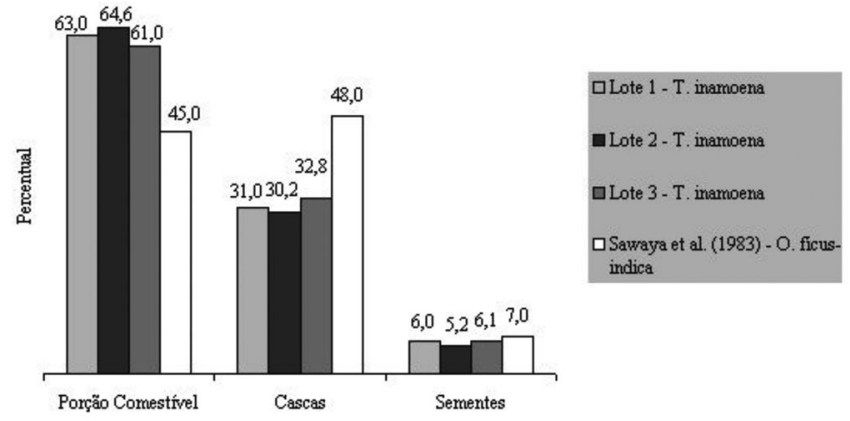

FIGURA 1 - Percentual de porção comestível, casca e sementes de frutos de T.inamoena e O. Ficus-indica

\section{CONCLUSÃO}

As características físicas, químicas e organolépticas de $T$. inamoena são similares às dos frutos da O. ficus-indica, usualmente consumidos in natura e industrializados. Dentre os constituintes de natureza nutricional determinados em $T$. inamoena, destacaram-se os minerais, principalmente o cálcio, o magnésio e o potássio. A semelhança entre as espécies, associada ao maior percentual de porção carnosa, constitui um indicador do potencial industrial do fruto, que pode ser explorado como alternativa alimentar e/ou como fonte de renda complementar para a agricultura familiar.

\section{REFERÊNCIAS}

ANDRADE LIMA, D. Plantas das caatingas. Rio de Janeiro: Academia Brasileira de Ciências, 1989. 243p.

AOAC - ASSOCIATION OF OFFICIAL ANALYTICAL CHEMISTRY. Official methods of analysis of the Association of Official Analytical Chemistry. $17^{\text {th }} \mathrm{ed}$. Washington: AOAC. 2002.

BARUFFALDI, R.; OLIVEIRA, M. N. Fundamentos de tecnologia de alimentos. São Paulo: Atheneu, 1998. 317p.

BICALHO, U. O.; PENTEADO, M. V. C. Estudo do fruto e do artículo da Opuntia ficus-indica (L) Miller cultivada em Valinhos-SP: I-Características bromatológicas. Revista de Farmácia e Bioquímica, São Paulo, v.17, n.1, p. 93-101, 1981.

BICALHO, U. O.; PENTEADO, M. V. C. Estudo do fruto e do artículo da Opuntia ficus-indica (L) Miller cultivada em Valinhos-SP: II-Características bioquímicas. Revista de Farmácia e Bioquímica, São Paulo, v.18, n.1, p. 68-74, 1982.

COELHO, R. R. P.; FERREIRA-NETO, C. J.; FIGUEIRÊDO, R. M. F.; QUEIROZ, A. J. M. Características físicas e físico-químicas do fruto da palma cultivado na região do Curimataú Paraibano. In:CONGRESSOBRASILEIRODECIÊNCIAETECNOLOGIA DEALIMENTOs, 19., 2004, Recife. Anais eletrônicos... 2004. CD-ROM.

GARCÍA, J. C.; VALDEZ, C. A. F. Nopalitos y tunas: producción, comercialización, poscosecha e industrialización. México: 
Chapingo, 2003.225p.

GLASS, V. Figo-da-índia: sabor entre espinhos. São Paulo. Maio, 2005. Seção Reportagens. Disponível em: <http:// globorural.globo.com/edic/185/rep figoa.htm $>$. Acesso em: 25 abril 2005.

INSTITUTO ADOLFO LUTZ. Normas analíticas do Instituto Adolfo Lutz: métodos químicos e físicos para análise de alimentos. 3. ed. São Paulo, 1985, v. 1.533p.

MARAIS, J. F. K.; ERASMUS, T. Body composition of Mugil cepholus, Liza dumerili, Liza richardsoni and Liza tricuspidens (Teleoste: Mugilidae) caught in the Swartkops estuary. Aquaculture, Londres, v. 10, n. 1, p. 75-86, 1977.

NERD, A.; MIZRAHI, Y. Biologia reprodutiva. In: BARBERA, G.; INGLESE, P. y PIMIENTA-BARRIOS, E. Agroecologia: cultivo e usos da palma forrageira. João Pessoa: SEBRAE, 2001. p.49-57. (FAO/ SEBRAE, 132).

RAMADAN, M. F.; MÖRSEL, J. Recovered lipids from prickly pear [Opuntia ficus-indica (L.)]peel: a good source of polyunsaturated fatty acids, natural antioxidant vitamins and sterols. Food Chemistry, Londres, v. 83, p. 447-456, 2003.

RODRIGUEZ-AMAYA, D.B. Aguide to carotenoids analysis in foods. Washington: Ilsi, 1999.119p.

SÁENZ, C. Processing technologies: an alternative for cactus pear (Oputia spp.) fruits and cladodes. Journal of Arid Environments, Londres, v. 46, p. 209-225, 2000.

SAWAYA, W. N.; KHATCHADOURIAN, H. A.; SAFI, W. M.; AL-MUHAMMAD, H. M. Chemical characterizacion of prickly pear pulp, Opuntia ficus-indica, and the manufacturing of prickly pear jam. Journal of Food and Technology, Oxford, v.18, p.183-193, 1983.

SAWAYA, W. N.; KHAN, P. Chemical characterization of prickly pear seed oil, Opuntia ficus-indica. Journal of Food Science, Chicago, v. 47, p. 2060-2061, 1982.

SEPÚLVEDA, E.; SÁENZ, C. Características químicas y físicas de pulpa de tuna (Opuntia ficus-indica). Revista de Agroquímica y Tecnología de Alimentos, Valência, v. 30, n. 4, p. 551-555, 1990.

STATSOFT. Statistic for Windows 6.0. Tulsa, 2001.

THÉ, P. M. P.; CARVALHO, V. D.; ABREU, C. M. P.; NUNES, R. P.; PINTO, N. A. V. Efeito da temperatura de armazenamento e do estádio de maturação sobre a composição química do abacaxi CV. Smooth Cayenne L. Ciências Agrotécnicas, Lavras, v. 25, n. 2, p.356-363, 2001.

VALDEZ, C.A.F. Produción y comercialización de la tuna. Reporter de Investigación, Chapingo, n. 67, dez. 2002. 